\title{
Endomarketing: una herramienta de integración del cliente interno con la es- trategia organizacional ${ }^{1}$
}

\section{Endomarketing: a tool for integration of internal customer with the organiza- tional strategy}

Lina Cristina Villa Gil ${ }^{2}$

lcvg7264@gmail.com

Darling Nathali Gómez Ospina ${ }^{3}$ darlingnathali@yahoo.es

\begin{abstract}
Resumen
Durante años se pensó que el cliente era solo un agente externo, objeto de un producto o servicio, pero se ha notado que los clientes existen tanto al interior como al exterior de la empresa y es entonces cuando se comienza a hablar del cliente interno, de tal forma que la preocupación por mantener y desarrollar el estado del cliente externo se extienda al mismo nivel del interno, debido a que el recurso humano representa el activo más valioso de toda organización y por ende este activo se debe conservar en aras de evitar la alta rotación de personal.
\end{abstract}

Debido a que la rotación de personal es un tema tan sensible para toda organización, el presente artículo presenta el Endomarketing como una herramienta de gestión para las organizaciones con el fin de integrar en su estructura estrategias para desarrollar y mantener el cliente interno, por lo cual se analiza su contexto, importancia, beneficios y dificultades con el fin de desarrollar una estrategia dirigida hacia el cliente interno dentro de la organización.

Palabras clave: Mercadeo; Endomarketing; Estrategia Organizacional; Recursos humanos.
Articulo de reflexión.

${ }_{2}^{2}$ Especialista en Gerencia del Talento Humano. Ejecutiva Metodológica del Banco W. ORCID https://orcid.org/0000-0003-3087-3913

${ }^{3} \mathrm{Mg}$. en Administración y Dirección de Empresas. Docente Uniminuto Bogotá Sur. ORCID https://orcid.org/0000-0002-2513-3382

${ }^{*}$ Cómo citar este artículo: Villa, L., Gómez, D. (2018). Endomarketing: una herramienta de integración del cliente interno con la estrategia organizacional. Hojas y Hablas, (15), 143-156. DOI: 10.29151/hojasyhablasn.15a9
For years it was thought that the client was only an external agent, the target of a product or service, but it has been noted that customers exist both within and outside the company, that is when speaking about the internal customer, in such a way that the concern to maintain and develop the condition of the external customer is worded at the same level of the internal one, since human resource is the most valuable asset of any organization and therefore this asset should be maintained in order to avoid the high staff turnover.

Due to the staff turnover is a sensitive issue for any organization, this article presents the Endomarketing as a management tool for organization that helps to integrate in its structure, strategies to develop and maintain the internal customer, such that context, importance, benefits and difficulties are analyzed in order to develop a strategy addressed toward the internal customer within the organization.

Keywords: Marketing; Endomarketing; Organizational Strategy; Human resource. 


\section{Introducción}

El presente artículo está basado en la Monografía de Grado presentada por parte de la estudiante Lina Christina Villa Gil para optar al título de Especialista en Gerencia del Talento Humano y se desarrolló bajo el tipo de investigación de orden descriptivo, exploratorio y bibliográfico, con el fin de conocer los principales postulados del Endomarketing enfatizados hacia mejores ambientes de trabajo.

En la actualidad generar mejores ambientes de trabajo no es una tarea fácil, la sociedad actual demanda nuevas ocupaciones y las empresas establecen sus prioridades en el cliente externo y es ahí donde los empleados empiezan su búsqueda de nuevas oportunidades laborales que les brinden mejores opciones de desarrollo profesional y cuyas empresas también implementan estrategias con el cliente interno, con lo cual se generan interrogantes alrededor del personal de una empresa que involucra empleados y empleadores, surgen preguntas como: ¿Qué hacer para fidelizar al empleado? ¿Cuáles son los mecanismos que ayudan a generar el interés del empleado por su empresa? ¿Se puede potenciar el sentido de pertenencia hacia la empresa? Pero, ¿qué hacer para asumir los retos que se presentan?, ¿cómo generar un producto de buena calidad y costo competitivo? ¿Cómo llamar la atención de nuestros clientes para conservarlos y fidelizarlos?

Para solucionar los problemas que implican éstos y otros interrogantes, se plantean estrategias productivas apoyadas en teorías administrativas y se implementan tendencias de servicio, de modo que el producto llegue con fuerza al cliente final.

"Está demostrado que aquellas compañías donde se presenta una alta rotación de personal, generan menor valor y están abocadas permanentemente a dificultades para competir" (Ardila, 2013), por lo cual se hace necesario implementar estrategias que detengan la rotación de personal o la fuga de talentos, generando competitividad en el entorno, promoviendo el reconocimiento de la organización en el mercado.

El Endomarketing surge entonces como una estrategia que busca fortalecer las relaciones al interior de la organización, involucrando a los colaboradores en la construcción de la cultura organizacional, aprovechando su creatividad y el conocimiento que tiene cada uno de ellos y que puede ser de gran aporte al logro de resultados. El Endomarketing está orientado a fidelizar, motivar e implicar a los empleados con su trabajo para generar sentido de pertenencia con la institución y evitar la deserción.

Esta situación se traduce en que los colaboradores se sienten parte de la empresa, perciben que sus necesidades e ideas son tenidas en cuenta y por ende la rotación de personal disminuye. Así mismo, la inversión en capacitación de nuevo personal disminuye, así como los gastos prestacionales de las liquidaciones del personal que se retira $y$, como se mencionó antes, se eliminan altos costos ocultos causados por la fuga de conocimiento.

Globalización, como factor principal de impulso de la integración económica colombiana con sus socios comerciales internacionales influyen directamente en que la competencia productiva sea cada vez más estrecha; tanto así, que solo aquellas compañías que han asimilado esta situación como oportunidades de crecimiento y mejora continua por los retos que se presentan, han sostenido y mejorado su statu-quo en la sociedad industrial de nuestro país. 
A través del tiempo, se ha enfocado la razón de ser de una compañía hacia la satisfacción del cliente final de su producto y en torno a ello se emplean las teorías de producción, teorías de administración y, muy especialmente, las teorías de marketing que estructuran la producción, comercialización y venta del mismo. Pero para poder vender una idea, un producto o un servicio, se debe creer en ellos, si se quiere que los clientes acojan el producto como lo mejor y más confiable para ellos, de modo que supla la necesidad que los aqueja.

Durante años se pensó que el cliente era solo este agente externo, objeto de nuestro producto, pero se ha notado que éste existe tanto al interior como al exterior de nuestra empresa; se comienza a hablar, entonces del cliente interno de tal forma que la preocupación por mantener el estado del cliente externo hace que se ponderen al mismo nivel.

Por tanto, si se implementa una herramienta como el marketing para atraer y sostener el cliente externo, se podrá adaptar esta herramienta para tratar de igual forma al cliente interno $y$ es allí donde se encuentra el tema sobre el que se fundamentara este trabajo: Endomarketing (Mercadeo Interno).

Es en este sentido que el Endomarketing toma fuerza como una herramienta efectiva para aplicar los mecanicismos de mejora. Esta es una estrategia que no solo debería tomarse como de mercadeo o comunicaciones sino como una estrategia integral de negocios que ayuda al fortalecimiento de la imagen corporativa tanto para los clientes internos como para los externos.

\section{Marco teórico}

La mercadotecnia durante el siglo XXI ha tomado participación en innumerables áreas, lo cual ha implicado su desarrollo teórico con elementos específicos como es el caso del Endomarketing o mercadeo interno, el cual considera estrategias dirigidas a los empleados; esta iniciativa se centra en la necesidad que tienen las organizaciones de sincronizar a todos sus trabajadores y/o colaboradores con la empresa y que sus acciones estén encaminadas al logro de los objetivos de la misma para mantener su motivación y buscar resultados positivos organizacionales. (Santamaria, 2017)

El término Endomarketing pareciera ser muy reciente en el lenguaje empresarial, pero en realidad desde finales del siglo pasado comienza a tomar fuerza el concepto. Sin embargo, el fundamento del Endomarketing ha sido aplicado por viejos empresarios en nuestro país. Tal es el caso de Adolfo Carvajal quien ha dejado un legado, el cual se tiene presente para que las empresas adopten estas políticas a sus empleados y desarrollen el cliente interno independientemente de su tamaño, del sector productivo al que pertenezcan, sean privadas o públicas.

El Endomarketing es el conglomerado de estrategias y acciones propias del marketing que se planean y ejecutan al interior de las organizaciones con el propósito de incentivar al personal. Se trata de motivar a los trabajadores, crear un mejor clima organizacional y obtener un mayor grado de lealtad y compromiso en la relación empresa - proveedor interno. (González \& Victorio, 2013, p 17) 
El entorno empresarial de hoy es cada vez es más agresivo, cambiante y veloz, lo cual exige a las empresas enfrentar mayores por lo que se ven abocadas a atraer y retener colaboradores productivos y eficientes que sepan afrontar la incertidumbre provocada por estos factores.

El objetivo del Endomarketing es el de satisfacer las necesidades y expectativas del cliente interno, de manera análoga a lo que hace el Mercadeo tradicional que busca fidelizar a los compradores o clientes externos reales y atraer a los potenciales.

Autores reconocidos como Rafiq y Ahmed (1993) argumentan las fases de construcción del concepto de marketing interno. La primera fase, marca el surgimiento del concepto, que fue caracterizada por la motivación y satisfacción del empleado. La segunda, estuvo orientada para el cliente externo, es decir, reconocía la importancia de la satisfacción del cliente interno en el alcance de los objetivos. La tercera fase, se refiere a la implantación y gestión de cambios organizacionales; en esta fase se describe el uso de las técnicas o herramientas de marketing para promover los cambios necesarios y mejorar la performance de la organización. Estos autores concluyen en la existencia de varios conceptos en relación al tópico, pero no hay unanimidad entre ellos.

El concepto de marketing interno está evolucionando, comienza a ser visto dentro de los mecanismos que permiten reducir fricciones interdepartamentales e interfuncionales con la finalidad última de vencer la resistencia al cambio en las organizaciones. Los nuevos aportes de la literatura académica han ido configurando el marketing interno como una filosofía de gestión, al igual que un instrumento general para la puesta en funcionamiento de cualquier estrategia organizativa, interna o externa (Alvarado, 2011).

Si la empresa quiere mantenerse vigente en el mundo empresarial de hoy, es recomendable que una de sus estrategias sea la de utilizar el Endomarketing para que los colaboradores acepten los desafíos motivados por el reconocimiento del valor de su trabajo por parte del nivel directivo, el establecimiento de objetivos claros y alcanzables, la satisfacción de sus necesidades y un clima laboral propicio para la creatividad y el desarrollo personal y profesional.

Hay que lograr que el colaborador trabaje con amor y pasión hacia la consecución de sus objetivos. Almustafá, el elegido y bien amado maestro de la magistral obra El Profeta escrita en 1923 por Khalil Gibrán, respondió así ante la pregunta ¿Qué es trabajar con amor?: "Es tejer la tela con hilos extraídos de vuestro corazón como si vuestro ser amado fuera a usar esa tela. Es construir una casa con cariño, como si vuestro ser amado fuera a habitar en esa casa" (Gibrán, 2003, p.12).

El mercadeo es diferente a ventas, publicidad, investigación y otros conceptos ligados a la gestión comercial. (American Marketing Association, 2012, prr 3) aprobó en julio de 2013 la definición de Mercadotecnia, como "La actividad, conjunto de instituciones y procesos para crear, comunicar, entregar, y el intercambio de ofertas que tienen valor para los clientes, los socios y la sociedad en general"

Esta definición conduce a destacar dos elementos fundamentales: la oferta de las instituciones y la demanda de los clientes para satisfacer sus necesidades. El mercadeo es el puente entre estos dos factores, por lo que se convierte en un dinamizador del mercado como 
herramienta para desarrollar las funciones administrativas de planear, organizar, direccionar, ejecutar y controlar las actividades gerenciales y empresariales.

(Villa,2013, prr6)afirmaqueel "Laformación de los mercados (clientes) se ha fundamentado siempre en un proceso de relaciones tendiente a lograr confianza mutua para tener una clientela fiel o leal. Las empresas familiares, y las pymes, como ocurre con las personas, deben hacer lo que esté al alcance de cada una para ello: sin grandes inversiones, pero actuando con lo que pueden y tienen. Si se quiere, como se dice, con las uñas, que también vale. “

El marketing juega un papel importantísimo en la vida actual porque gracias a todas sus actividades desarrolladas con los agentes en la economía, las personas podemos disfrutar de los bienes necesarios para satisfacer las necesidades y mantener nuestro nivel de vida. (López, 2005, parr. 2)

El mercadeo podría asemejarse como la estructura social creada alrededor del intercambio de bienes o servicios; es toda una red en pro de satisfacer una necesidad, un deseo o incluso para generarlos.

Las organizaciones construyen sus filosofías de mercadeo con el objetivo de conquistar un mercado, de modo que lleve su producto a niveles rentables que generen crecimiento y auto sostenibilidad.

El marketing es un proceso social y de gestión a través del cual los distintos grupos e individuos obtienen lo que necesitan y desean, creando, ofreciendo e intercambiando productos con valor hacia otros. La gestión de marketing es una filosofía de la organización al servicio al cliente con el fin de satisfacer sus necesidades y lograr los objetivos de la empresa. El concepto de marketing implica acción al momento de conquistar los mercados, análisis para comprenderlos y una ideología en la construcción de la sociedad de consumo, por lo cual, la gerencia de marketing debe cumplir responsabilidades como el análisis de las oportunidades, establecimiento de objetivos, organización, recursos para la mezcla, creación de la oferta y evaluación y control de actividades de marketing.

La gestión de marketing es una organización destinada al servicio del cliente para la satisfacción de sus necesidades y lograr los objetivos de supervivencia, rentabilidad y crecimiento, se pretende desarrollar, fidelizar y mantener a cada cliente.

El marketing es un sistema de acción que realiza una serie de actividades en la economía basadas en el intercambio y analiza las fuerzas del mercado, la demanda y la oferta. Tiene fundamentos ideológicos como lo son los medios de venta que utiliza para conquistar mercados; mediante la publicidad, las promociones de ventas y la venta personal. Con los análisis y estudios de los mercados se enfoca a las necesidades y a la demanda de los consumidores y con su ideología genera actitudes hacia el consumo.

Los objetivos estratégicos de mercadeo son los logros fundamentales que se desean alcanzar para garantizar el crecimiento de una empresa o áreas de la misma, en el corto, mediano y largo plazo.

"El marketing influye en los deseos y la demanda haciendo atractivo el producto, propone medios para satisfacer las necesidades, define la 
forma de los productos, el posicionamiento, la diferenciación, las acciones promocionales y los estilos de vida" (López, 2005, párr. 2).

Partiendo de lo planteado por José Ariel Giraldo, el marketing es un proceso mediante el cual se identifican las diferentes necesidades del ser humano y se diseñan productos o servicios con características específicas para la satisfacción de las mimas. De igual forma, uno de sus objetivos principales es la fidelización (retención) del cliente, ya que en el mediano plazo esto le garantiza a la empresa rentabilidad y sostenibilidad.

La aplicación del Mercadeo depende de muchas variables exógenas, lo que hace que esta actividad sea compleja y complicada, aunque se comprenda muy bien su teoría. Ninguna estrategia de mercadeo aplicada en una empresa puede ser desarrollada exactamente igual en otra semejante, debido a que los productos no son réplicas exactas unos de otros, los responsables de esta función son diferentes, los ambientes son distintos, las condiciones guardan sus diferencias. Lo que sí puede hacerse es analizar la estrategia de mercadeo en una empresa a la luz de las necesidades particulares de otra para adaptarla a su propia realidad.

Si la gestión del marketing está orientada al servicio al cliente mediante la satisfacción de sus necesidades y deseos, la gestión del Endomarketing debe dirigirse al cliente interno para garantizar el cumplimiento de los objetivos del marketing empresarial. Es necesario fidelizar al cliente interno para lograr la fidelización del cliente externo.

"Sin ventas no hay salvación" dice un refrán empresarial, pero para lograr el nivel de ventas que permita alcanzar los objetivos de la empresa es necesario definir estrategias de marketing tradicional y no tradicional, por cuanto la tecnología ha cambiado la forma de llegar al cliente y al consumidor final.

El éxito del marketing, depende en gran medida del conocimiento del individuo; es decir, en la implementación de estrategias, se debe partir del hecho fundamental de la diferenciación, pues, aunque las necesidades son esencialmente las mismas, las prioridades cambian de acuerdo a las personas.

Pero si bien el marketing es un proceso social y de gestión, la clave no está solo en acertar con las estrategias definidas, sino que es necesario un trabajo duro y persistente con cada una de ellas, analizar sus resultados, corregir los defectos que se vayan encontrando y no parar en buscar el elemento diferenciador que va a destacar a la empresa por encima de su competencia. El mejor marketing es el trabajo duro, ya que es poco probable que se consiga algo a cambio de no hacer nada o muy poco.

\section{Importancia del mercadeo}

Las empresas han cambiado sus enfoques debido a la globalización, avances de la ciencia, la obsolescencia de los productos, cambios en los valores de la sociedad y las nuevas tecnologías comerciales. Desde la ventaja competitiva, se hace necesario mejorar más la calidad en el servicio al cliente, ofrecerle mayores beneficios, menores precios, crear más valor, mantener mayores vínculos sociales, manteniendo personal orientado al cliente que no sean de horario y que disfruten de su trabajo y lo vean como algo retador y gratificante, es decir colaboradores con altos niveles de motivación y compromiso con el cliente y la organización. 
Es necesario fomentar el trabajo en equipo, la colaboración, la cooperación y el apoyo conjunto y el alto desempeño de los distintos departamentos de las empresas para mantener para mantenerse en función del cliente" (López, 2005, párr.2).

La actividad del Mercadeo ha sido cuestionada por muchos sectores con razón o sin razón, entre estas, la falta de lealtad de los clientes, que prima hoy día, el abuso de la publicidad con ciertos mensajes subliminales y el desempeño de algunos vendedores. Es por ello que algunos directivos han tenido al Mercadeo como algo secundario en sus empresas y, en el mejor de los casos, lo "utilizan" para vender sus productos o servicios.

El mercadeo, como cualquier otra función en la empresa, debe entenderse como un trabajo de seres humanos para seres humanos, buscando siempre el bienestar de todos: cliente interno, cliente externo y la sociedad en general.

\section{Endomarketing}

La palabra Endomarketing tiene un uso reciente en el argot de la administración y, más concretamente, en el área de gestión humana. La palabra está compuesta de dos elementos: el primero endo, prefijo griego que significa interno; el segundo, marketing que traduce mercadeo. Por lo tanto, Endomarketing se entiende como la estrategia empresarial orientada a favorecer la fidelización del cliente interno de la empresa, tiene como principal objetivo generar el sentido de pertenencia entre sus colaboradores.

En la década del 70, la reconocida compañía Johnson \& Johnson atravesaba por una crisis interna que obligó a su director, Saúl Bekin, a pensar en una estrategia que generara en sus colaboradores una actitud de compromiso con la empresa. Es así como decide "vender" la empresa a sus empleados de la misma manera que se hace con los clientes externos; surgió entonces el concepto de Mercadeo Interno o Endomarketing.

\section{Levionnois (1992) afirma que:}

el marketing interno es, ante todo, considerar la gestión y la optimización de los recursos humanos como una finalidad en sí misma, y no como uno de los medios puestos al servicio de la empresa para alcanzar con más seguridad los objetivos de rentabilidad.

Esta es la base del Endomarketing, el cual se combina con el Desarrollo Organizacional que propende por establecer procesos de cambio y de evolución de la empresa partiendo de favorecer la actitud de cambio y de compromiso del colaborador.

El Endomarketing tiene un impacto alto $y$ directo en la cultura organizacional ya que ésta está compuesta por el conjunto de valores y creencias esenciales de la empresa. Recoge el comportamiento individual y colectivo, los valores o su ausencia, el lenguaje propio, el estilo gerencial y organizacional, el ambiente laboral y el clima organizacional. Todo lo anterior es generado por el individuo. Debe recordarse que la calidad de una sociedad está en relación directa con la calidad de los individuos que la componen; así mismo, la calidad de una empresa está determinada por la calidad de sus colaboradores, como lo afirma (González, Sánchez \& López, 2011, p. 17). El Endomarketing busca que los empleados se sientan parte de la empresa, que tomen conciencia de la importancia de sus aportes al fortalecimiento de la misma y que lleven a cabo acciones que redunden en un mayor beneficio y mejores resultados para 
la organización y para ellos mismos.

Desde una perspectiva general el Endomarketing está orientado a generar motivación en los colaboradores, de tal manera que impacte en la satisfacción de los stakeholders o partes interesadas. Para lograr este resultado, la empresa debe trabajar en el bienestar laboral y un clima organizacional adecuado, de tal manera que se brinde al colaborador las condiciones más favorables para que su productividad esté siempre en los niveles necesarios para cumplir con los objetivos finales de la organización; en este sentido, el Endomarketing se vuelve estratégico por cuanto fortalece las capacidades del empleado.

Por otro lado, cuando el Endomarketing se utiliza para llevar a cabo campañas dirigidas a demostrar que para la empresa los colaboradores son reconocidos como el pilar fundamental y son el objetivo principal de la organización, la consecuencia lógica son los resultados económicos. Esta perspectiva convierte al Endomarketing en Táctico.

Cualquiera sea la perspectiva del Endomarketing, siempre buscará cumplir con el objetivo principal: La motivación del colaborador a través de su mejoramiento permanente de la calidad de vida laboral.

\section{El Endomarketing como herramienta de desarrollo organizacional}

El ser humano siempre será un artesano en tanto adopte la actitud de aprendiz de la vida, sin importar su clase social ni su nivel de conocimiento. El directivo empresarial no se excluye de esta situación entendiendo que el elemento principal de la empresa es el colaborador a quien se le debe entregar todas las posibilidades de desarrollo personal y profesional.
(Alles, 2011, p. 51) Para un exitoso programa de desarrollo profesional de las personas se deben tener en cuenta los conocimientos requeridos por la posición que la persona ocupa en el presente o cualquier otra que eventualmente pueda asumir en el futuro

Por tanto, el Desarrollo Organizacional centra su actividad en el conocimiento de las relaciones humanas en la empresa, lo cual conlleva un trabajo de la gerencia a fin de que los colaboradores encuentren un sentido real a la labor que realizan en ella.

Se observa cómo el Endomarketing y el Desarrollo Organizacional guardan una relación directa. El Endomarketing se basa en aspectos comola motivación, la satisfacción del colaborador, el clima laboral y la cultura organizacional, lo que se constituye en la materia prima para adelantar procesos de Desarrollo Organizacional.

Los procesos de cambio organizacional deben adelantarse de manera tal que se elimine la mayor cantidad posible de rechazo, derivado del temor y del confort que representa mantenerse en unas condiciones determinadas. Al implementar estrategias organizacionales, la empresa puede llegar a una situación de conflicto interno ya que los empleados pueden verse obligados a cambiar esquemas de comportamiento, o a sentirse amenazados por nuevas estructuras organizacionales, o por nuevas políticas, procesos o procedimientos, entre otros aspectos.

El Endomarketing, funciona como un instrumento de acción, destacando la forma en que los empleados construyen una cultura al interior de una organización. Por lo cual, es esencial diferenciar los elementos diferenciadores en la realización de estrategias de Endomarketing 
en las empresas, tomando como punto de partida el sector económico en el que desarrollan su actividad principal, las áreas de desempeño y la ejecución de acciones del marketing interno, el plan de incentivos y la fidelización de los empleados.

Aplicando los principios del Endomarketing se busca generar mejores relaciones entre la satisfacción de los clientes externos y el cliente interno; implementando estrategias de marketing que logren retener a los talentos de las organizaciones y apuntando a una nueva dimensión de la Gestión del Talento Humano. Para llegar a los resultados esperados, las empresas deberán adoptar una filosofía de gestión estratégica que le permita hacer una mejor administración de su principal recurso, los colaboradores, apoyándose el marketing interno. Del éxito de la implementación de esta herramienta, se conseguirá impactar de forma positiva al cliente externo y se logrará llegar a la generación de una ventaja competitiva frente a los pares de la organización.

Es fundamental lograr el sentido de pertenencia en los colaboradores, pues finalmente son el puente entre el sentimiento y la vivencia que conlleva a la asimilación, la pasión, el entendimiento y el compromiso con lo que hacen.

Para generar sentido de pertenencia, deben existir impulsos motivacionales, bien sea generados por sí mismo, por otras personas o por las circunstancias que le rodean y que exigen la satisfacción de necesidades humanas.

Un directivo de una empresa debería reflexionar y hacerse éstas y otras preguntas del mismo orden:

- ¿Cómogenerarenlos colaboradores dela organización el sentido de pertenencia hacia la empresa?

- ¿Cuáles son los impulsos motivacionales que se establecen?
- ¿Cuáles son lasnecesidades de nuestros colaboradores que satisfacemos? ¿Cuáles no?

Para generar sentido de pertenencia es necesario descubrir si las personas que se encuentran bajo nuestra responsabilidad viven orientadas hacia la superación de los retos y obstáculos, mediante lo cual buscan su desarrollo y su crecimiento como personas y profesionales (no solamente desde el punto de vista académico) o, por el contrario, son perfeccionistas y necesitan realizar trabajos que exigen gran calidad y quieren desarrollar sus habilidades y destrezas.

Tal vez sean de aquellas afectivas que requieren relacionarse con los demás en un medio social y que necesitan de la palmadita en el hombro o la felicitación cuando han tenido acciones positivas. No hay que olvidar que también hay personas con ambiciones de poder, dominadas por el deseo imperante de influir en los otros y que incluso están dispuestos a arriesgar al máximo para alcanzarlo.

Nunca se estará rodeado por personas que pertenezcan a un solo perfil, por esta razón, el éxito de la gestión, está en descubrir la faceta de cada uno, lo que depende de la ubicación del directivo frente a sí mismos; en otras palabras, se requiere conocer cuáles son los factores motivacionales que generan el propio sentido de pertenencia y cómo éstos se relacionan con los demás.Beneficios y dificultades del Endomarketing

Si bien una de las principales ventajas del Endomarketing es obtener la coordinación entre las diferentes áreas de la empresa, sin interferir con el adecuado desarrollo e implementación de las estrategias organizacionales; cabe destacar que cuenta con otros beneficios tales como: 
- Promover el trabajo en equipo, permitiendo espacios donde los colaboradores puedan expresar sus ideas. Esto conlleva a evitar fricciones entre las personas y reduce el riesgo del fracaso; ya que, al existir comunicación entre las partes, hace posible evidenciar de manera oportuna los diferentes obstáculos.

- De otra parte, con la integración de todas las áreas, se incrementan las posibilidades de viabilidad de las estrategias propuestas.

Aun así, y como en todo proceso de implementación de estrategias, también puede traer consigo dificultades que entorpecen el objetivo de las mimas. En el Endomarketing, algunas de los conflictos más comunes son:

- Apatía por parte de los colaboradores, producto del desconocimiento que tienen acerca de proyectos y actividades que realiza la empresa ya que no se le proporciona esta información oportunamente.

- De lo anterior se desprende otro aspecto a negativo como lo es la falta de criterio y autonomía, ya que el colaborador no tiene claro cuál es el impacto de su rol dentro de la organización y esto genera falta de compromiso.

- Producto de esto, el colaborador no desarrolla sentido de pertenencia ni lealtad hacia la empresa; limitándose a cumplir con el trabajo asignado, sin generar ideas o iniciativas que proyecten las bondades que tiene la empresa hacia el cliente externo.

Aunque el Endomarketing es una disciplina con resultados significativos, todavía no cuenta con una amplia proyección, quizás por desconocimiento, resistencia al cambio, carencia de recursos $o$ desinterés a involucrar nuevas disciplinas en los procesos de planeación estratégica organizacional.

Actualmente las empresas desarrollan y aplican manuales de acción, donde pretenden abarcar grandes volúmenes de trabajo que generan procesos mecánicos, los cuales se transforman en hábitos para los empleados; y esto a su vez, genera problemas de comunicación interna en el largo plazo, debido a que la información obtenida viaja en una misma dirección, ocasionando confusión de acciones y la presencia de elementos distractores como el ruido, las emociones desequilibradas, la desconfianza, el trabajo bajo presión, la incoherencia de acción y reacción, y por último la imposición de objetivos y metas organizacionales (Dávila, 2013, p. 12)

Por lo anterior, si las empresas esperan obtener resultados exitosos, con competitividad y eficiencia, es preponderante que comiencen por implementar estrategias enfocadas, en primera instancia, en el cliente interno, con el fin de evitar la deserción, incrementar el sentido de pertenencia y aprovechar al máximo el conocimiento que poseen sus colaboradores.

En el desarrollo de estas estrategias, los líderes del proceso podrían tener en cuenta iniciativas como:

- Procesos de capacitación constante, donde exista el intercambio de roles con el propósito de que cada una de las personas involucradas en el proceso conozcan más acerca la importancia del cargo que desempeña cada uno dentro de la organización. Esta iniciativa conlleva a que se incremente el trabajo en equipo y por ende a generar un mejor clima laboral.

- Comprobar el hecho que los colaboradores cuenten con la confianza por parte de sus superiores para ser autónomos en la toma de decisiones relacionadas directamente con su trabajo, sin 
comprometer su buen funcionamiento.

- Que el colaborador conozca el detalle de cuáles son los aspectos tenidos en cuenta por la empresa cuando se llevan a cabo las evaluaciones de desempeño.

\section{Importancia del Endomarketing}

El desarrollo de compromisos es fundamental en toda empresa, cada empleado constituye una pieza de vital importancia para la organización que forma un todo para la función substancial de la misma, por lo cual las compañías en la actualidad realizan actividades para formar empleados íntegros, que a su vez generen buen clima organizacional, trabajo en equipo y que se sincronicen como una unidad productiva dentro de la organización, siempre con el compromiso individual de crecer tanto personal como profesionalmente.

El tener una gran motivación, permite establecer relaciones humanas satisfactorias de animación, interés y colaboración, cuando la motivación es escasa, ya sea por frustración o por impedimentos para la satisfacción de necesidades, el clima organizacional tiende a dañarse $y$ nacen estados de depresión, desinterés, apatía, descontento, hasta llegar a estados de agresividad e inconformidad, característicos de situaciones en que los empleados se enfrentan abiertamente contra la empresa como son los casos de huelgas, mítines, entre otros. (Fuentes, 2012, p. 49).

Cuando un cliente externo se siente satisfecho con el producto o servicio que recibe, habitualmente vuelve a adquirirlo, además en cuanto haya una oportunidad de recomendarlo, no tendrá reparo en hacerlo. De igual manera, ocurre con un colaborador que se siente motivado, implicado y satisfecho con su trabajo, pero en este caso, la ganancia que está obteniendo la empresa es que cuenta con un cliente interno comprometido y con una alta tendencia de orientación al resultado.

Cuando los colaboradores se sienten implicados, comprometidos y automotivados, esto se traduce en lealtad y deseos de permanecer en la empresa, lo cual está directamente relacionado con la disminución en los indicadores de rotación de personal y por ende, en el incremento de la rentabilidad, pues con esto, se reducen los costos de capacitación de nuevo personal y los gastos por prestaciones que se desprenden de la liquidación de los empleados que renuncian. Sin dejar de lado, que se está evitando con ello, otro factor, no menos importante como lo es la fuga de conocimiento.

Para consolidar equipos de trabajo implicados, es necesario cerciorarse de que, a los colaboradores de forma individual, les gusta lo que hacen con el fin generar enlaces emocionales que inciten al liderazgo positivo y proactivo. Para llevar a cabo estos vínculos, tienen que existir espacios donde se mantenga la comunicación constante entre los colaboradores y se hagan reconocimientos a las buenas iniciativas.

El sentido de pertenencia está estrechamente ligado con el concepto de implicar, ya que, en ambos casos, el objetivo final es propiciar la conexión humana entre el cliente interno y el externo, situación que se traducirá en éxito empresarial. Para el ser humano es esencial que se le trate como tal, no como simples medios para la consecución de resultados; por esta razón, si se propenden por mejores relaciones entre los individuos, el resultado se obtendrá de forma más eficiente y perdurable en el tiempo.

De la misma manera, la implicación le apuesta a explorar la creatividad e innovación de los colaboradores, pues está demostrado que las 
empresas con mejores resultados económicos, son aquellas donde existen altos niveles de compromiso y experiencias innovadoras. Por esto, es necesario tener en cuenta que, para incentivar y potenciar la creatividad del colaborador, se requiere generar espacios de independencia con el fin de concebir un entorno de seguridad que incite a crear.

\section{Componentes de la estrategia del Endomarketing}

Lacomunicación por medio deherramientas como la intranet, carteleras informativas, revistas corporativas, reuniones, entre otros; se hacen con el fin de que el colaborador (cliente interno) mantenga informado de los aspectos importantes de la empresa y que les concierne de manera directa o indirecta, en su desarrollo profesional dentro de la compañía.

Cuando se habla de integración, se refiere al hecho de que todos y cada uno de los colaboradores que hacen parte de la empresa, es un pilar importante en la evolución de la misma.

Y la motivación está enfocada en el conocimiento del colaborador y los aspectos de la vida que le motivan con el fin de generar estrategias que le permitan el desarrollo de sus expectativas.

El Endomarketing propone llevar a cabo actividades en las cuales lo colaboradores se integren y compartan entre sí, y de ser posible, involucrando familias con el objeto de generar experiencias que marcan de forma positiva y que estrechan a la empresa con el vínculo emocional más difícil de medir, pero a su vez, el que más aviva la motivación y el compromiso del colaborador.
De la adecuada implementación, gestión, aplicación y evaluación de la herramienta del Endomarketing dependerá, en gran medida, el generar en los colaboradores, sentimientos de compromiso y sentido de pertenencia, resultado del grado de motivación de cada uno de ellos, es un beneficio que va en doble vía pues a mayor grado de satisfacción con la calidad de vida laboral, mayor será el compromiso que tiene el colaborador frente a las funciones asignadas dentro de la empresa, reflejado de forma directa y positiva en el cliente externo, y por ende, en los resultados económicos de la organización

\section{Conclusiones}

El Endomarketing es la herramienta por medio de la cual se puede llegar a construir un puente entre la empresa y el colaborador, donde convergen las expectativas y objetivos de cada una de las partes mencionadas; lo cual es el punto de partida para la generación de estrategias que integren al colaborador con los objetivos estratégicos de la organización, pero donde también la empresa propende por el bienestar de sus colaboradores, a través de procesos que incrementen la motivación del mismo.

El éxito del Endomarketing dependerá en gran medida de la estrategia implementada por la empresa, la ejecución realizada y el seguimiento que se le realice con el fin de analizar los resultados $y$ sus alcances para el beneficio de todos los empleados.

Aunque el Endomarketing tiene su enfoque en la satisfacción de las necesidades del cliente interno, tiene una orientación directa hacia el cliente externo, lo cual genera en los colaboradores un sentimiento de compromiso para con la empresa $\mathrm{y}$ sus clientes finales. 
El Endomarketing es una gestión estratégica del área de Gestión Humana desde el enfoque del marketing donde la diferencia es que para el primero su mercado objetivo está al interior de la organización, es decir, el talento humano, el pilar sobre el cual se fundamenta la estrategia.

El Endomarketing es una herramienta de gran valor para las organizaciones porque condensa el potencial de los colaboradores, desde la generación de espacios donde se sientan valorados, motivados y comprometidos con los objetivos de la organización, lo que a su vez, convierte a la empresa en un ente económico altamente productivo, competitivo y rentable; además de socialmente responsables, dado que está orientada a los pensamientos, sentimientos y emociones de su recurso más importante; el talento humano.

\section{Referencias}

Alles, M. (2007). Desarrollo del Talento Humano, basadoencompetencias. Recuperadodehttp:// saludpublica.bvsp.org.bo/textocompleto/ bvsp/boxp68/talento-humano_a.pdf

Alvarado, S. (15 de noviembre de 2011). Gestiopolis. Recuperado de Gestiopolis: http://www.gestiopolis.com/marketing/plande-marketing-interno.html

American Marketing Association. (1 de octubre de 2012). Recuperado de American Marketing Association: https://www.marketingdirecto. com/punto-de-vista/la-columna/31definiciones-de-mercadotecnia-9
Ardila, H. (2013). www.elempleo.com.co. Recuperado de Rotación de Personal, un enemigo Potencial: http://www.elempleo. com/colombia/noticias_laborales/ rotacin-n-de-personal-un-enemigopotencial-/6586754

Dávila, S. (2013). La Satisfacción del cliente interno a través del Endomarketing. Recuperado de http://repository.ean.edu.co/bitstream/ handle/10882/3831/DavilaAna2013. pdf? sequence $=1$

Fuentes, S. (2012). Satisfacción laboral y su influencia en la productividad. Quetzaltenango, Guatemala.

Gibrán, K. (2003). El Profeta. Recuperado de http://www.biblioteca.org.ar/libros/11402. pdf

González, Sánchez \& López. (2011). La importancia del Marketing Interno en las Organizaciones. Management y Empresa, 6.

González, E. \& Victorio. (octubre de 2013). Merca 20. Recuperado de http://merca20.com

Levionnois, M. (1992). Marketing interno y gestión de recursos humanos, Traducción de Les éditions dórganizatión (1987). Madrid: Díaz.

López, J. A. (16 de febrero de 2005). Gestiopolis. com. Recuperado de Gestiopolis.com: https://www.gestiopolis.com/teoria-delmercadeo/

Rafiq, M. \& Ahmed. (1993). The scope of Internal marketing: defining the boundary between marketing and human resource management. Journal of Marketing 
Management, 219-232.

Santamaria, A. (2017). El Endomarketing y la satisfacción del cliente interno de la empresa Importadora, Alvaro Vásconez Cia Ltda de la ciudad de Ambato. Recuperado de http:// repo.uta.edu.ec/bitstream/123456789/2659 8/1/599MKT.pdf

Villa, C. F. (2013). El mercadeo en las Pymes.
Recuperado de Tiempodemercadeo: http://www.tiempodemercadeo.com/ articulos-de-mercadeo/373-el-mercadeode-las-pymes

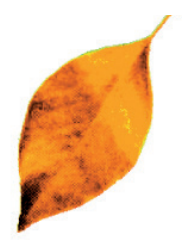

